\title{
Моделирование режимов ручейкового безнапорного течения жидкой фазы рабочего тела в элементах абсорбционных холодильных аппа- ратов
}

\author{
А. С. Титлов ${ }^{1 凶}$, О.Б. Васылив ${ }^{2 凶}$, Д.Б. Адамбаев \\ Одесская национальная академия пищевых технологий, ул. Канатная, 112, Одесса, 65039, Украина \\ ORCID: ${ }^{1}$ 0000-0003-1908-5713; ${ }^{2}$ 0000-0002-0597-8863 \\ $\rrbracket^{1}$ e-mail: titlov1959@gmail.com; $\rrbracket^{2}$ e-mail: oleg_vas@ukr.net; $\rrbracket^{3}$ e-mail: adambayev90@gmail.com
}

\begin{abstract}
При переходе бытовой холодильной технике на природные рабочие тела особое место занимают холодильники с абсорбционными холодильными агрегатами (АХА). Основной недостаток абсорбционных холодильников высокое энергопотребление. Это связано с отсутствием модельных представлением о процессах тепломассообмена и режимов течения рабочего тела в элементах АХА при разработке и проектировании серийньх моделей. Рассмотрень силь, действующие на элемент жидкого хладагента, движущегося равномерно внутри гладкой трубы. Приведена модель течения без учета поверхностного натяжения жидкости. Применялось два способа решения поставленной задачи. При первом способе предполагалось, что течение жидкости - слоистое и ее слои располагаются конщентрично внутренней поверхности трубы. Второй способ решения поставленной задачи также предполагает слоистость течения жидкости, однако, без ограничения по расположению слоев. Приведена модель расчета течения с учетом сил поверхностного натяжения. Принято, что криволинейная поверхность раздела фаз представляет собой поверхность круглого цилиндра радиуса.
\end{abstract}

Ключевые слова: абсорбционные холодильные агрегаты, ручейковое безнапорное течение, моделирование, расчет, анализ

\section{Моделювання режимів струмкової безнапірної течії рідкої фази робо- чого тіла в елементах абсорбційних холодильних апаратів}

\section{О. С. Тімлов, О. Б. Василів, Д. Б. Адамбаєв}

Одеська національна академія харчових технологій, вул. Канатна, 112, Одеса, 65039, Україна

\begin{abstract}
При переході побутової холодильної техніки на природні робочі тіла особливе місие займають холодильники з абсорбиійними холодильними агрегатами (АХА). Основний недолік таких холодильників - високе енергоспоживання. Це пов'язано з відсутністю модельних уявлень про прочеси тепломасообміну і режими течї̈ робочого тіла в елементах АХА при розробиі і проектуванні серійних моделей. Розглянуто сили, щзо діють на елемент рідкого холодоагенту, щьо рухається рівномірно всередині гладкої труби. Наведено модель течії без урахування поверхневого натягу рідини. Застосовувалося два способи вирішення поставленого завдання. При першому способі передбачалося, щзо течія рідини - шарувата і їі шари розташовуються концентрично внутрішньої поверхні труби. Другий спосіб вирішення поставленого завдання також передбачає наявність шарів течії рідини, однак, без обмеження по розташуванню шарів. Наведено модель розрахунку течії з урахуванням сил поверхневого натягу. Прийнято, щуо криволінійна поверхня розділу фаз є поверхнею круглого ичиліндра радіуса.
\end{abstract}

Ключевые слова: абсорбционные холодильные агрегаты, ручейковое безнапорное течение, моделирование, расчет, анализ

(C) The Author(s) 2018. This article is an open access publication

This work is licensed under the Creative Commons Attribution 4.0 International License (CC BY) http://creativecommons.org/licenses/by/4.0/

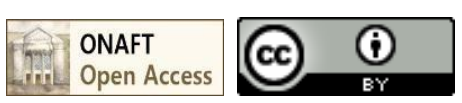

\section{I. ВВЕДЕНИЕ}

При переходе бытовой холодильной технике на природные рабочие тела особое место занимают холодильники с абсорбционными холодильными агрегатами (АХА). Рабочее тело АХА - водоаммиачный раствор с инертным газом (водородом, гелием либо их смесью [1]). Работа абсорбционных холодильников может осуществляться от любого источника тепловой энергии с достаточным температурным потенциалом [2]. АХА не имеют движущих элементов в конструкции, бесшумны и надежны в работе, стоимость их минимальна среди аналогов [3].

Основной недостаток абсорбционных холодильников - высокое энергопотребление.

Во многом это связано с отсутствием модельных представлением о процессах тепломассообмена и ре- 
жимов течения рабочего тела в элементах АХА при разработке и проектировании серийных моделей.

И если есть некоторые разработки в части моделирования и расчета процессов тепломассообмена в элементах AXA $[4,5]$, а в том числе и в парогазовом контуре [6], то до настоящего времени должного внимания процессам гравитационного течения жидкой фазы рабочего тела не уделялось.

Известна лишь модель расчета испарителя АХА, в которую входят эмпирические соотношения, заимствованные из работ Ленинградского института пищевой и холодильной промышленности и позволяющие определить среднюю скорость течения жидкой фазы и по ней конфигурацию сечения ручейка [7].

Эти соотношения, однако, применимы только к трубам с гладкой внутренней поверхностью, они не учитывают влияния сил поверхностного натяжения, искривляющих межфазную поверхность; кроме того, для этих не приведен диапазон параметров, в котором они получены. Поэтому их следует рассматривать, как ориентировочные и применимые лишь для предварительных оценок.

В специальной литературе [8] рассматриваются методы расчета (на основе коэффициентов Шези) ручейковых турбулентных сечений, характерных для речных стоков и каналов больших размеров, что не применимо для труб испарителя и абсорбера АХА с диаметрами порядка 20 мм.

\section{II. ОСНОВНЫЕ ПОЛОЖЕНИЯ}

Многочисленные экспериментальные и аналитические исследования сотрудников Одесской национальной академии пищевых технологий и Васильковского завода холодильников [9-12] показали, что в цилиндрической трубе испарителя АХА имеет место ламинарное течение испаряющейся жидкой фазы хладагентааммиака. При этом сечение трубы жидкостью занято лишь частично (рис. 1).

Жидкость образует ручеек, перемещающийся под действием гравитации вдоль и вниз по трубе, расположенной под углом к горизонту. Такое течение жидкости в гидравлике именуется безнапорным ручейковым течением [8]. Аналогичное течение имеет место и в абсорбере AXА, где жидкой фазой является водоаммиачный раствор.

Важность правильного определения параметров течения и конфигураций поперечных сечений фаз при расчете и проектировании испарителей и абсорбера АХА, определило задачу настоящего исследования как разработку модели безнапорного ручейкового течения жидкости в трубах небольшого диаметра (до 30 мм).

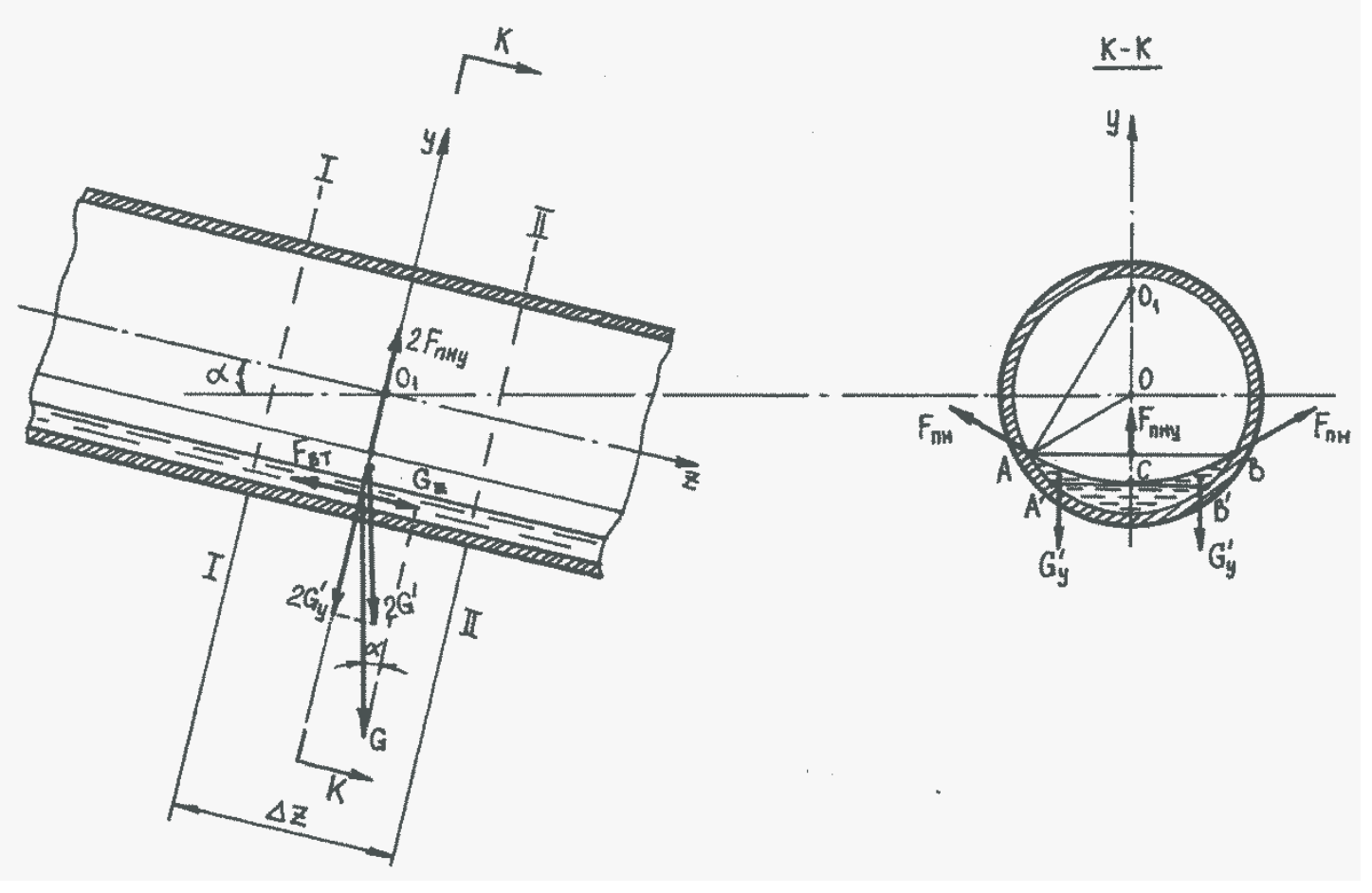

Рисунок 1 - Силы, действующие на элемент жидкости на длине $\Delta Z$ при безнапорном ручейковом течении жидкости в круглой трубе

Рассмотрим силы, действующие на элемент жидкого хладагента, движущегося равномерно внутри гладкой трубы между сечениями I-I и II-III, расстояние между которыми равно $\Delta Z$ (рис. 1). При этом жидкий хладагент смачивает поверхность трубы. На него действуют: вес $G$, направленный вертикально вниз; равнодействующая сил вязкостного трения $F_{\text {вm }}$, направленная против движения, и две равнодействующие сил поверхностного натяжения $F_{n н}$, направленные по касательной к поверхности раздела АСВ нормально к линиям контакта жидкости с поверхностью трубы.

Условия равномерности движения вдоль оси выразится равенством сил

$$
G \cdot \sin \alpha=F
$$


В предположении о неизменности конфигурации сечения элемента жидкости при течении проекция равнодействующей обеих сил поверхностного натяжения $\Sigma F_{n н y}$ на ось $y$ должна уравниваться проекцией на эту же ось веса $\Sigma G_{y}^{\prime}$ жидкости в объеме, ограниченном поверхностью раздела АCВ, и горизонтальной плоскостью А'C'B'. Таким образом

$$
\Sigma G_{y}^{\prime}=\Sigma F_{n н y}
$$

На основе уравнений (1) и (2), выражающих баланс сил, приложенных к элементу жидкости, будем решать поставленную задачу. Первоначально не будем учитывать поверхностное натяжение жидкости.

\section{III. МОДЕЛЬ ТЕЧЕНИЯ БЕЗ УЧЕТА ПОВЕРХНО- СТНОГО НАТЯЖЕНИЯ ЖИДКОСТИ}

При таком подходе уравнение (2) отсутствует, и решение может быть получено на основе уравнения (1). Поверхность раздела фаз в этом случае плоская и поперечное сечения трубы выглядит, как это показано на рис. 2.

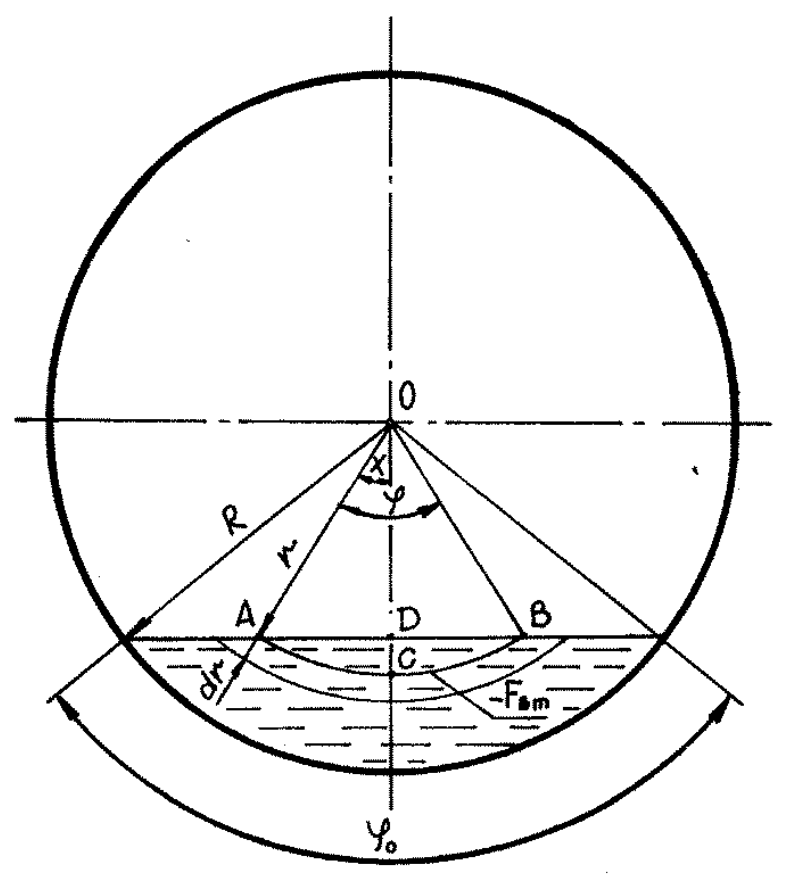

Применялось два способа решения поставленной задачи. При первом способе предполагалось, что течение жидкости - слоистое и ее слои располагаются концентрично внутренней поверхности трубы. В потоке жидкости между сечениями I-I и II-III выделялся слой ADBCA, ограниченный плоской поверхностью раздела фаз ADB и цилиндрической, концентричной внутренней поверхности трубы, поверхностью АСВ с текущим радиусом r, определяющимся, как это следует из геометрии, формулой

$$
r=\frac{R_{o} \cdot \cos \frac{\varphi_{o}}{2}}{\cos \frac{\varphi}{2}},
$$

где $R_{o}$ - радиус внутренней поверхности трубы;

$\varphi_{o}$ - центральный угол, охватывающий сегмент полного сечения;

$\varphi$ - текущее значение этого центрального угла. (Углы $x=\varphi / 2$ и $x_{o}=\varphi_{o} / 2$ введены для удобства выкладок).

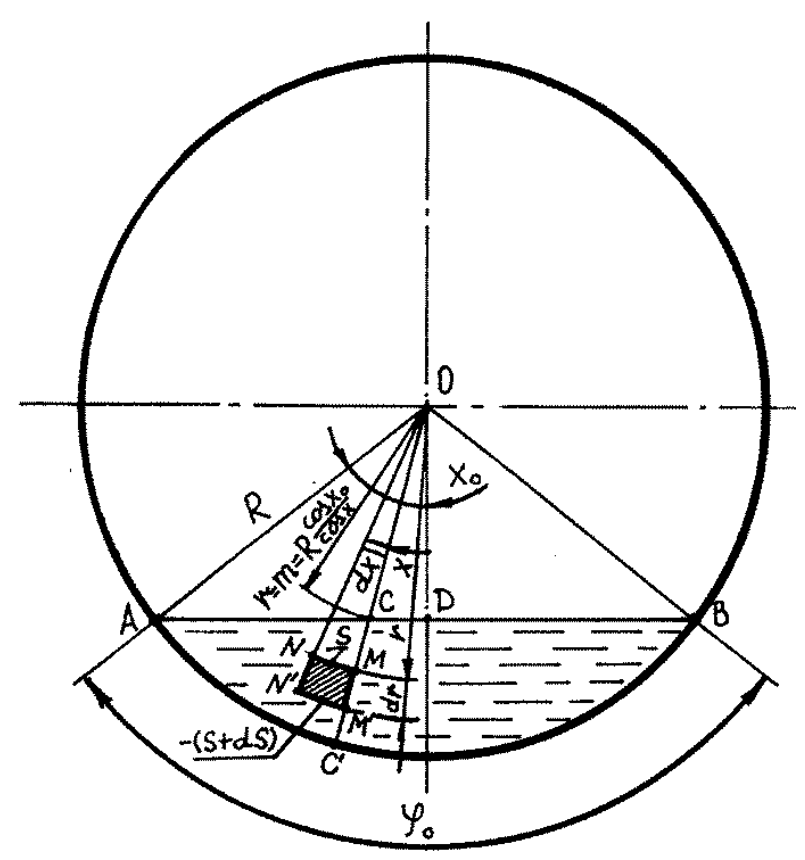

Рисунок 2 - Расчетные схемы для определения параметров безнапорного ручейкового ламинарного течения жидкости в круглой трубе (без учета поверхностного натяжения)

a - слои жидкости концентричны трубе; б - слои жидкости не концентричны трубе

Из рис. 1 и 2а следует, что для выделенного слоя левая (“весовая”) часть уравнения (1) запишется как

$$
G_{z}=g \cdot \rho_{\varkappa} \cdot f_{c e \varphi} \cdot \Delta Z \cdot \sin \alpha
$$

где $g$ - ускорение силы тяжести;

$\rho_{ж}$ - плотность жидкости;

$f_{\text {сеч }}$ - площадь поперечного сечения слоя (сегмента $\mathrm{ADBCA})$ - рис. 2a, составляющая

$$
f_{\text {cеч }}=\frac{r^{2}}{2} \cdot(\varphi-\sin \varphi)
$$

Трением между жидкостью и газом на поверхности раздела фаз $\mathrm{ADB}$, по сравнению с трением между слоями жидкости по поверхности АСВ, пренебрегаем. Тогда сила $F_{B T}$, действующая на выделенный слой со 
стороны следующего кольцевого слоя толщиной $d r$, определится (на основании соотношения Ньютона) как

$$
F_{\text {вm }}=-\mu_{ж} \cdot \frac{d W}{d r} \cdot f_{A C B}
$$

где $\mu_{ж}$ - коэффициент динамической вязкость жидкости;

$$
\frac{d W}{d r} \text { - градиент скорости; }
$$

$f_{A C B}$ - площадь цилиндрической поверхности, по которой осуществляется трение, равная

$$
f_{A C B}=r \cdot \varphi \cdot \Delta Z,
$$

где произведение $(r \cdot \varphi)$ - длина дуги АСВ.

Знак «минус» в (6) означает, что сила $F_{\text {вm }}$ направлена против течения.

В результате совместного рассмотрения (1), (3) - (7) уравнение (1) перепишется в виде

$$
\begin{aligned}
& g \cdot \rho_{\varkappa} \cdot \sin \alpha \cdot\left(R \cdot \cos x_{o}\right)^{2} \cdot \frac{2 x-\sin 2 x}{2 \cos ^{2} x}= \\
& =-2 \mu_{\varkappa} \cdot x \cdot r \cdot \frac{d W}{d r}
\end{aligned}
$$

На основании связи (3) производную $\left(\frac{d W}{d r}\right)$ можно выразить через соотношение $\left(\frac{d W}{d x}\right)$ как

$$
\frac{d W}{d r}=-A \cdot\left(\frac{\sin x}{\cos 3 x}-\frac{\operatorname{tg}^{2} x}{x}\right)
$$

В результате совместного рассмотрения (9) и (8) и соответствующих преобразований дифференциальное уравнение (8) приводится к виду

$$
W_{(x)}=\frac{A}{2} \cdot\left\{\left(\operatorname{tg}^{2} x_{0}-\operatorname{tg}^{2} x\right)-\left(x_{0}^{2}-x^{2}\right) \cdot\left[1+\frac{1}{3} \cdot\left(x_{0}^{2}+x^{2}\right)\right]\right\}
$$

Полученное выражение представляет собой зависимость скорости течения жидкости от половинного угла охвата сечения или (в силу соотношения (3)) от радиуса слоя.

Наибольшая скорость течения, очевидно, имеет ме-

$$
\frac{d W}{d r}=\frac{d W}{d x} \cdot \frac{\cos ^{2} x}{R \cdot \cos x_{o} \cdot \sin x}
$$

в котором

$$
A=\frac{g \cdot \rho_{\varkappa} \cdot \sin \alpha \cdot\left(R^{2} \cdot \cos x_{o}\right)^{2}}{2 \mu_{\varkappa}}
$$

Уравнение (10) может быть проинтегрировано как

$$
W=-A \cdot\left[\int \frac{\sin x}{\cos ^{3} x} d x-\int \frac{\operatorname{tg}^{2} x}{x} d x+C\right]
$$

Первый интеграл в выражении (12) - табличный и составляет

$$
\int \frac{\sin x}{\cos ^{3} x} d x=\frac{\operatorname{tg}^{2} x}{2}+C_{1}
$$

Второй интеграл не табличный и может быть взят после разложения $\operatorname{tg} x$ в ряд. Выбрав число членов ряда, обеспечивающих точность результатов в пределах $1 \%$, получим

$$
\int \frac{\operatorname{tg}^{2} x}{x} d x \approx \frac{x^{2}}{2} \cdot\left(1+\frac{x^{2}}{3}\right)+C_{2}
$$

Постоянную интегрирования в (12) $C=C_{1}+C_{2}$ найдем из условия: при равенствах $x=x_{0}, W=0$, так как у поверхности трубы скорость жидкостей практически отсутствует.

$$
C=\frac{A}{2} \cdot\left[\operatorname{tg}^{2} x_{0}-x_{0}^{2} \cdot\left(1+\frac{x_{0}^{2}}{3}\right)\right]
$$
ности трубы, т.е. в точке D (рис. 2a). Ей соответствует условие $x=0$ (или $r=R \cdot \cos x_{0}$ ), применив второе к (16) и учтя (11), для максимальной скорости получим сто

$$
W_{\max }=\frac{g \cdot \rho_{\varkappa} \cdot \sin \alpha \cdot R^{2}}{8 \mu_{\varkappa}} \cdot\left[\left(1-\cos \varphi_{0}\right)-\frac{\varphi_{0} \cdot\left(1+\cos \varphi_{0}\right)}{4} \cdot\left(1+\frac{\varphi_{0}^{2}}{12}\right)\right]
$$

где $\varphi_{0}$ - полный угол раскрытия сечения.

Уравнение (16) позволяет определить среднюю (по расходу жидкости) скорость течения, выражающуюся на основании уравнения сплошности как

$$
\bar{W}=\frac{\int_{0}^{f_{c e u}} W_{(x)} \cdot d f_{c e u}}{f_{c e u_{0}}},
$$

равная

$$
f_{\text {ceu }_{0}}=\frac{R^{2}}{2} \cdot\left(\varphi_{0}-\sin \varphi_{0}\right)
$$

Продифференцировав (5) с учетом (3) по величине $d \varphi$, получим 


$$
d f_{c e u}=a^{2} \cdot \frac{\varphi \cdot \sin \varphi}{(1+\cos \varphi)^{2}} \cdot d \varphi=2 a^{2} \cdot \frac{x \cdot \sin x}{\cos ^{3} x} \cdot d x
$$

$a=R_{0} \cdot \cos x_{0}$

Рассмотрев совместно (16), (18)-(21), после преобразований получим

где

$$
\begin{aligned}
& \bar{W}=\frac{2 A \cdot a^{2}}{R^{2} \cdot\left(\varphi_{0}-\sin \varphi_{0}\right)} \cdot\left[2 C \cdot \int_{0}^{x_{0}} x \cdot \operatorname{tg} x \cdot \sec ^{2} x \cdot d x-\int_{0}^{x_{0}} x \cdot \operatorname{tg}^{3} x \cdot \sec ^{2} x \cdot d x+\right. \\
& \left.\int_{0}^{x_{0}} x \cdot \operatorname{tg} x \cdot \sec ^{2} x \cdot d x+\frac{1}{3} \cdot \int_{0}^{x_{0}} x^{5} \cdot \operatorname{tg} x \cdot \sec ^{2} x \cdot d x\right]
\end{aligned}
$$

Интегралы, стоящие в выражении (22) - не табличные. Они брались путем соответствующего разложения в ряд функций $\operatorname{tg} x$ и $\sec x$ при ограничении числа членов ряда, обеспечивающем точность в пределах $3 \%$.

В результате интегрирования, соответствующих подстановок и преобразований, для средней скорости течения окончательно получено следующее выражение:

$$
\begin{aligned}
& \bar{W}=\frac{g \cdot \rho_{\varkappa} \cdot \sin \alpha \cdot R^{2} \cdot \varphi_{0}^{3} \cdot\left(1+\cos \varphi_{0}\right)^{2}}{96 \mu_{\varkappa} \cdot\left(\varphi_{0}-\sin \varphi_{0}\right)} \cdot\left\{\left(1+\frac{\varphi_{0}^{2}}{5}\right) \cdot\left[\frac{1-\cos \varphi_{0}}{1+\cos \varphi_{0}}-\frac{\varphi_{0}}{4} \cdot\left(1+\frac{\varphi_{0}^{2}}{12}\right)\right]+\right. \\
& \left.+\frac{\varphi_{0}^{4}}{80} \cdot\left[2.43 \cdot \frac{1-\cos \varphi_{0}}{1+\cos \varphi_{0}}-0,607 \varphi_{0}^{2} \cdot\left(1+\frac{\varphi_{0}^{2}}{12}\right)-\varphi_{0}^{2} \cdot\left(0,343+0,131 \varphi_{0}^{2}\right)-0,714\right]\right\}
\end{aligned}
$$

Средняя скорость течения жидкости, с другой стороны, может быть выражена через массовый расход $M_{x a}$ жидкости как

$$
\bar{W}=\frac{2 M_{\varkappa}}{\rho_{\varkappa} \cdot R \cdot\left(\varphi_{0}-\sin \varphi_{0}\right)}
$$

При заданном численном значении $M_{ж}$, на входе в испаритель или абсорбер, известном из термодинамического расчета цикла АХА (либо для последующего участка трубы, известного из расчета предыдущего), в результате решения системы (23)-(24) может быть определена средняя скорость течения $\bar{W}$ и угол $\varphi_{0}$, определяющий площадь и конфигурацию сечения.

С помощью полученных соотношений был проведен расчет параметров течения жидкого аммиака на входе в испаритель для цикла AXА при холодильной мощности агрегата 50 Вт.

В качестве исходных данных при расчете было принято [14]: плотность $\rho_{\text {э }}=671 \mathrm{~m} / \kappa г ;$ коэффициент динамической вязкости $\mu_{\text {ж }}=23,23 \cdot 10^{-6}$ Па $\cdot$; массовый расход $M_{\varkappa}=5,3 \cdot 10^{-5} \mathrm{\kappa r} / \mathrm{c} ;$ внутренний радиус трубы испарителя R=9,5 мм; угол наклона к горизонту $\alpha=2$.

В результате расчетов получено: раскрытие $\varphi_{0}=36,2 ; \quad \bar{W}=0,043 \mathrm{~m} / \mathrm{c} ; \quad \bar{W}_{\text {мах }}=0,075 \mathrm{~m} / \mathrm{c}$. Соответствующее поле скоростей представлено на рис. $3 \mathrm{a}$.

Как и следовало ожидать, при изложенном выше способе решения поставленной задачи линии постоянной скорости представляют собой дуги окружностей, концентрических внутренней поверхности трубы испарителя.
Возможен другой способ решения поставленной задачи, также предполагающий слоистость течения жидкости, однако без ограничения по расположению слоев.

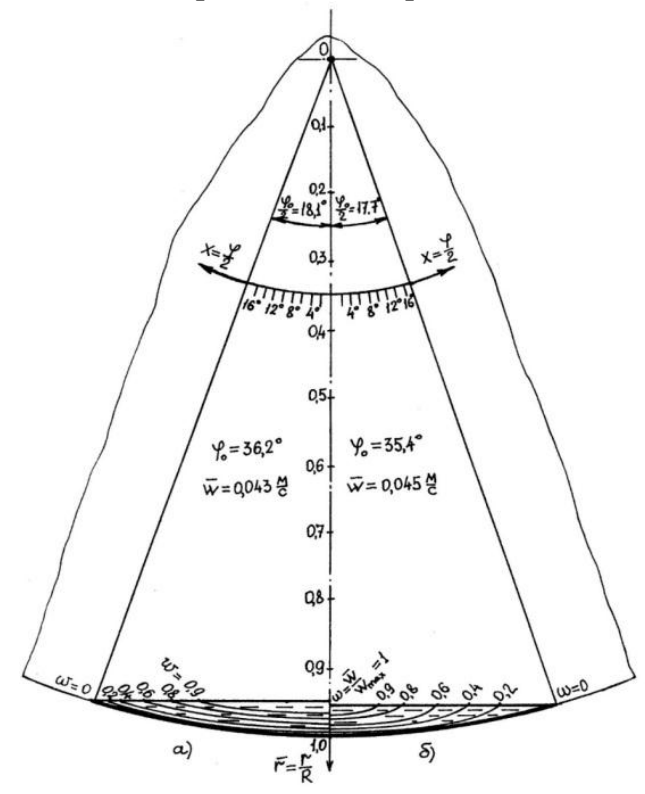

Рисунок 3 - Поля скоростей при безнапорном ручейковом ламинарном течении хладагента на входе в трубу испарителя AXА (без учета поверхностного натяжения): а - слои жидкости концентричны трубе; б - слои жидкости не концентричны трубе;

$$
\begin{aligned}
& Q_{0}=50 \mathrm{Bm} ; M_{\varkappa}=5,3 \cdot 10^{-5} \kappa 2 / \mathrm{c} ; \alpha=2^{\circ} ; R \\
& =9,5 \mathrm{MM} ; \rho_{\varkappa}=671 \kappa 2 / \mathrm{M}^{3} ; \mu_{\varkappa}=23,23 \cdot 10^{-5} \Pi a \cdot \mathrm{c}
\end{aligned}
$$


При таком способе решения задачи скорость потока жидкого аммиака в точке сечения $M$ определяется полярными координатами этой точки: радиусом $r$ и углом поворота $X$ (рис. 2б), изменяющихся в пределах:

$$
\begin{aligned}
& r=O C \ldots O C^{\prime}=R \frac{\cos x_{0}}{\cos x} \ldots R, \\
& x=0 \ldots x_{0}
\end{aligned},
$$

Выделим элементарный объем жидкости, ограниченный расположенными друг от друга на расстоянии $d r$, цилиндрическими поверхностями $N M_{\text {и }} N^{\prime} M^{\prime}$; плоскостями $O N^{\prime}$ и $O M^{\prime}$, пересекающимися под углом $d x$ и плоскостями сечений I-II и II-II (рис. 1), расположенным друг от друга на расстоянии $d z$.

На жидкость, заключенную в этот объем, действуют согласно рис. 1 элементарные силы $-d G_{z}$ (весовая составляющая) и $d F_{\text {вm }}$ (внутреннего трения).

Согласно (1) запишем

$$
d G \cdot \sin \alpha=d F_{\text {sm }}
$$

где элементарный вес жидкости составит

$$
d G=g \cdot \rho_{\varkappa} \cdot d f \cdot d Z,
$$

в котором площадь элементарного сечения $N M M^{\prime} N^{\prime}$ при пренебрежении бесконечно малыми 2-го порядка, равна

$$
d f=\left\{\pi \cdot\left[(r+d r)^{2}-r^{2}\right]\right\} \cdot \frac{d x}{2 \pi} \approx r \cdot d r \cdot d x
$$

Равнодействующая сил вязкостного трения, действующая на часть слоя жидкости, заключенную в элементарный объем $N M M^{\prime} N^{\prime}$, является суммой сил вязкостного трения, действующих на элементарные поверхности: $N^{\prime} M^{\prime}$, равную $[(r+d r) \cdot d x] \cdot d z$ и $N M$, равную $(r \cdot d x) \cdot d z$.

Учтя это и пренебрегая бесконечно малыми 3-го порядка, получим

$d F_{\text {вm }}=-(S+d S) \cdot(r+d r) \cdot d x \cdot d z+S \cdot r \cdot d x \cdot d z=$ $=-(S+d S) \cdot(r \cdot d x \cdot d z+d r \cdot d x \cdot d z)+S \cdot r \cdot d x \cdot d z \approx$ $\approx-d S \cdot(r \cdot d x) \cdot d z$

где $d z$ - элементарное изменение силы удельного вязкостного трения в слое $d r$

Из совместного рассмотрения (26)-(29) получим

$$
g \cdot \rho_{\varkappa} \cdot \sin x=-\frac{d S}{d r}
$$

Из формулы Ньютона для вязкости трения $S=\mu_{\varkappa} \cdot\left(\frac{d W}{d r}\right)$ следует

$$
\frac{d S}{d r}=\mu_{\varkappa} \cdot \frac{d^{2} W}{d r^{2}},
$$

что после подстановки в (30) приводит к дифференциальному уравнению 2-го порядка в виде

$$
\frac{d^{2} W}{d r^{2}}=B
$$

где

$$
B=-\frac{g \cdot \rho_{\varkappa} \cdot \sin \alpha}{\mu_{\varkappa}}
$$

Проинтегрировав (32) первый раз в пределах $O C=m$ и $O M=r$ (рис. 2) получим

уравнение 1-го порядка

$$
\frac{d W}{d r}=-B \cdot\left(R \cdot \frac{\cos x_{0}}{\cos x}-r\right),
$$

величина текущего радиуса $m=R \cdot \frac{\cos x_{0}}{\cos x}$, а точка $C$ (конец текущего радиуса) лежит на межфазной г(ð)рхности, на которой $\frac{d W}{d r} \approx 0$, ввиду того, что трением между жидкостью и газом, по сравнению с трением между слоями жидкости, пренебрегаем.

Проинтегрировав (34) в пределах $O M=r \quad$ и $O C^{\prime}=R$, что при условии $O C^{\prime}=R \quad W=0$, после преобразований получим соотношение:

$$
\begin{aligned}
& W_{(x, r)}=\frac{g \cdot \rho_{\varkappa} \cdot \sin \alpha}{\mu_{ж}} \cdot(R-r) . \\
& {\left[R \cdot\left(\frac{1}{2}-\frac{\cos x_{0}}{\cos x}\right)+\frac{r}{2}\right]}
\end{aligned}
$$

выражающие зависимость скорости жидкости в точке его поперечного сечения от полярных координат этой точки $r$ и $x$.

Выражение для максимальной скорости (в точке сечения D) получим из (35) при условии $x=0$ и $r=R \cdot \cos x$. Это выражение преобразований приобретет вид

$$
W_{\text {max }}=\frac{g \cdot \rho_{\varkappa} \cdot \sin \alpha \cdot R^{2}}{2 \mu_{\varkappa}} \cdot\left(1-\cos x_{0}\right)^{2}
$$

Среднюю скорость течения жидкости будем, как и при первом способе решения задачи, определять на основе соотношения (18), которая при данном способе запишется как

$$
\bar{W}=\frac{2 \int_{0}^{x_{0}} \int_{R \cdot \frac{\cos x_{0}}{\cos x}}^{R} W_{(x, r)} \cdot r \cdot d r \cdot d x}{f_{0}}
$$




$$
\bar{W}=\frac{g \cdot \rho_{\varkappa} \cdot \sin \alpha \cdot R^{2}}{\mu_{\varkappa} \cdot\left(\varphi_{0}-\sin \varphi_{0}\right)} \cdot\left\{\frac{\varphi_{0}}{4}+\cos \frac{\varphi_{0}}{2} \cdot\left[\left(\frac{1+\cos \varphi_{0}}{2}\right) \cdot \ln \left(\operatorname{tg}\left(\frac{\pi+\varphi_{0}}{4}\right)\right)-\frac{1}{3} \ln \left(\frac{1+\sin \frac{\varphi_{0}}{2}}{1-\sin \frac{\varphi_{0}}{2}}\right)\right]-\right.
$$

$$
\left.-\frac{5}{36} \cdot \sin \varphi_{0} \cdot\left(2+\cos \varphi_{0}\right)\right\}
$$

В результате двойного интегрирования (37) с учетом (35), преобразований и соответствующих подстановок для средней скорости окончательно получим (38).

Решая (38) совместно с (24), можно определить при заданных расходе жидкости $M_{x a}$ и радиусе трубы $\mathrm{R}$ среднюю скорость его течения и угол $\varphi_{0}$, определяющий площадь и конфигурацию сечения.

На рис. $3 б$ приведено поле скоростей и характеристики течения: $W=0,045 \mathrm{~m} / c ; \varphi_{0}=35,4^{\circ}$, полученные с помощью соотношений (35) и (37) для тех же условий, что и для первого способа.

Из рисунка видно, что слои жидкости не концентричны внутренней поверхности трубы, и тем в большей степени, чем они от нее удалены. Этого и следовало ожидать исходя из качественно-интуитивных представлений о ручейковом течении.

Численно средняя скорость и конфигурация сечения при обоих способах определения отличаются незначительно (в пределах 5 \%). Поэтому для практических расчетов могут применяться оба способа. При этом, следует учитывать, что расчетные соотношения по первому способу получены с точностью до 3 \% в диапазоне $\varphi_{0}=0 \ldots \frac{\pi}{2}$, так как интегрирование (22) осуществлялось при соответствующем расположении в ряд тригонометрических функций, в то время как аналогичные соотношения по второму способу получены без таких ограничений ввиду того, что интегрирование (37) сводилось к взятию табличных интегралов.

\section{IV. МОДЕЛЬ РАСЧЕТА ТЕЧЕНИЯ С УЧЕТОМ СИЛ ПОВЕРХНОСТНОГО НАТЯЖЕНИЯ}

Модель будем строить на основе уравнений баланса сил (1) и (2). При этом будем предполагать, что криволинейная (в результате поверхностного натяжения жидкости) поверхность раздела фаз АCDB представляет собой поверхность круглого цилиндра радиуса $R_{2}$ (рис. 4). В этом случае конфигурация сечения будет определяться не только центральным углом $\varphi_{0}$, но и максимальной толщиной потока $\delta_{0}$. Из геометрических соображений и соответствующих выкладок следует, что эти величины связаны между собой следующим образом

$$
\begin{aligned}
& R_{2}=\frac{2 R \cdot\left(R-\delta_{0}\right) \cdot\left(1-\cos x_{0}\right)+\delta_{0}^{2}}{2\left[R \cdot\left(1-\cos x_{0}\right)-\delta_{0}\right]}, \\
& \text { где } x=\frac{\varphi_{0}}{2}
\end{aligned}
$$

\section{Силы поверхностного натяжения $F_{n н}$, действую-} щие по линиям $A$ и $B$ контакта жидкости с трубой нормально к этим линиям и по касательной к поверхности раздела фаз (рис. 1) и (рис. 4), составляют $\sum F_{n н}=2 \delta_{\text {ж }} \cdot \Delta Z$, а их проекция на ось $y$ выразится как

$$
\sum F_{n H y}=2 \delta_{\varkappa} \cdot \Delta Z \cdot \sin x_{2}^{0}
$$

так как угол $\angle A \cdot F_{n t} \cdot F_{n x y}$ равен углу $\angle A O_{1} O$ и равен углу $\angle x_{2}^{0}$ (углы с соответственно перпендикулярными сторонами), где $x_{2}^{0}$ - половина центрального угла, охватывающего сечение потока по окружности $R_{2}$; здесь он характеризует краевой угол смачивания $\theta$, являясь $x_{2}=\frac{\pi}{2}-\theta ; \sigma_{ж}$ - коэффициент поверхностного натяжения жидкости.

К этому же соотношению можно прийти и другим путем - через дополнительное, связанное с поверхностным натяжением, давление на жидкость, в результате которого возникает разность уровней между точками $A(B)$ и точкой $D$. Это дополнительное давление по закону Лапласа [13] составляет

$$
\Delta P=2 \delta_{\varkappa} \cdot \bar{K},
$$

где $\bar{K}$ - средняя кривизна поверхности по двум главным взаимно-перпендикулярным направлениям. В нашем случае одно из них нормально к оси потока, другое - параллельно ей.

В соответствии с этим

$$
\bar{K}=\frac{1}{2}\left(\frac{1}{R_{2}}+\frac{1}{\infty}\right)=\frac{1}{2 R_{2}},
$$

где радиус кривизны в нормальном к оси направлении равен

$$
R_{2}=\frac{b}{2 \sin x_{2}^{0}},
$$

в котором $b$ - хорда сечения потока.

Это дополнительное давление, действуя на уровне точек $A$ и $B$ на прямоугольную площадку $(b \cdot \Delta Z)$, вызывает дополнительную силу $F$, уравновешиваемую весом жидкости в объемах $A D A^{\prime}$ и $D B B^{\prime}$, и равную в соответствии с (41) - (43) 


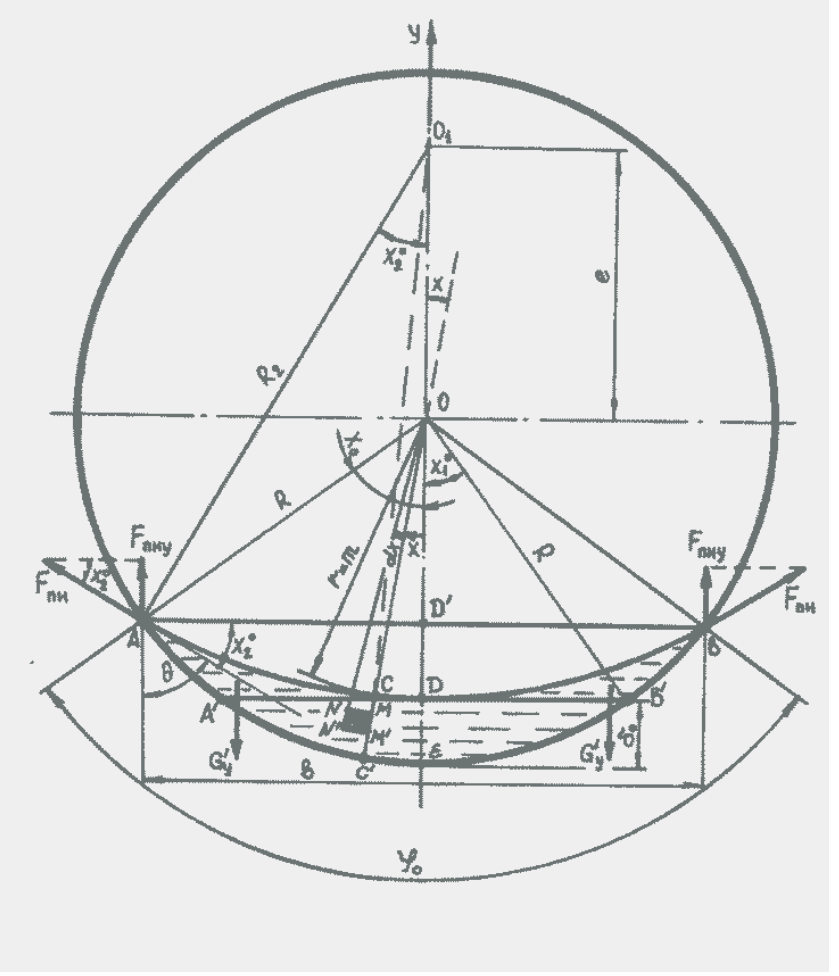

Рисунок 4 - Расчетная схема для определения параметров безнапорного ручейкового течения жидкости в круглой трубе (с учетом поверхностного натяжения

$$
F=\frac{\delta_{\varkappa}}{R_{2}} \cdot(b \cdot \Delta Z)=2 \delta_{\varkappa} \cdot \Delta Z \cdot \sin x_{2}^{0}
$$

которая, согласно (40), совпадает с силой $\sum F_{n н y}$, т.е. является соответствующей проекцией равнодействующей сил поверхностного натяжения.

Сумма весов жидкости, заключенной в одинаковых объемах $A D A^{\prime}$ и $D B B^{\prime}$ составит $\sum G^{\prime}=g \cdot \rho_{ж} \cdot \Delta F \cdot \Delta Z$, а ее проекция на ось $y$ (рис. 1) выразится как

$$
\sum G_{y}{ }^{\prime}=g \cdot \rho_{\varkappa} \cdot \Delta F \cdot \Delta Z \cdot \cos \alpha
$$

в котором суммарная площадь сечений $A D A^{\prime}$ и $D B B^{\prime}$ равна

$$
\Delta F=f_{A D^{\prime} B E A}-f_{A D^{\prime} B D A}-f_{A^{\prime} C B^{\prime} E A^{\prime}},
$$

где величины, стоящие в правой части представляют собой площади соответствующих сегментов.

На основе выражений (40) и (46) уравнение баланса сил (2) примет вид

$$
2 \delta_{\varkappa} \cdot \sin x_{2}^{0}=g \cdot \rho_{ж} \cdot \cos \alpha \cdot \Delta F,
$$

в котором для приращения площади $\Delta F$ из геометрических соображений получено

$$
\Delta F=R^{2} \cdot\left\{\left[\left(x_{0}-x_{1}^{0}\right)-\sin \left(x_{0}-x_{1}^{0}\right) \cdot \cos \left(x_{0}+x_{1}^{0}\right)\right]-\left(\frac{\sin x_{0}}{\sin x_{2}^{0}}\right)^{2} \cdot\left(x_{2}^{0}-\frac{\sin 2 x_{2}^{0}}{2}\right)\right\},
$$

где

$$
x_{1}^{0}=\arccos \left(1-\frac{\delta_{0}}{R}\right)
$$

Угол $x_{2}^{0}$, на основе равенства с учетом (39), может быть выражен как

$$
\begin{aligned}
& \frac{4 \delta_{\varkappa} \cdot \sin x_{0} \cdot\left[R \cdot\left(1-\cos x_{0}\right)-\delta_{0}\right]}{R_{1} \cdot g \cdot \rho_{\varkappa} \cdot \cos \alpha \cdot\left[2 R \cdot\left(R-\delta_{0}\right) \cdot\left(1-\cos x_{0}\right)+\delta_{0}^{2}\right]}= \\
& =\left\{x_{0}-\arccos \left(1-\frac{\delta_{0}}{R}\right)-\sin \left[x_{0}-\arccos \left(1-\frac{\delta_{0}}{R}\right)\right] \cdot \cos \left[x_{0}+\arccos \left(1-\frac{\delta_{0}}{R}\right)\right]\right\}- \\
& -\arcsin \left(\frac{2 R \cdot \sin x_{0} \cdot\left[R \cdot\left(1-\cos x_{0}\right)-\delta_{0}\right]}{2 R \cdot\left(R-\delta_{0}\right) \cdot\left(1-\cos x_{0}\right)+\delta_{0}^{2}}\right)- \\
& -\frac{1}{2} \sin \left\{2 \arcsin \left(\frac{2 R \cdot \sin x_{0} \cdot\left[R \cdot\left(1-\cos x_{0}\right)-\delta_{0}\right]}{2 R \cdot\left(R-\delta_{0}\right) \cdot\left(1-\cos x_{0}\right)+\delta_{0}^{2}}\right)\right\} \cdot\left\{\frac{2 R \cdot\left(R-\delta_{0}\right) \cdot\left(1-\cos x_{0}\right)+\delta_{0}^{2}}{2 R \cdot\left[R \cdot\left(1-\cos x_{0}\right)-\delta_{0}\right]}\right\}^{2}
\end{aligned}
$$

Уравнение (51) выражает при прочих равных условиях связь между геометрическими параметрами сече- ния потока $x_{0}$ и $\delta_{0}$ при учете поверхностного натяжения жидкости. 
При применении уравнения движения (1) в случае поверхностного натяжения рассмотрим, как и ранее, силы, действующие на жидкость, заключенную в элементарный объем $N M M^{\prime} N^{\prime}$ в направлении оси $z$ (рис. 1 и 4). Это те же силы - весовая составляющая $d G_{z}$ и сила внутреннего трения $d F_{s m}$, для которых также справедливы соотношения (26) - (29) и полученное на их основе дифференциальное направление 2-го порядка (32).

Проинтегрировав (32) первый раз в тех же пределах, получим

$$
\frac{d W}{d r}=-B \cdot(m-r)
$$

Однако, при учете поверхностного натяжения вели чина текущего радиуса $O C=m$ выражается достаточно сложной геометрической зависимостью от текущего значения угла $x$, что связано с кривизной поверхности раздела фаз. Получим эту зависимость, рассмотрев треугольник $C O \mathrm{O}$. По теореме косинусов запишем для него соотношение

$\left(O_{1} C\right)^{2}=(O C)^{2}+\left(O O_{1}\right)^{2}-2(O C) \cdot\left(O O_{1}\right) \cdot \cos \angle O_{1} O C$ из которого учтя, что $O_{1} C=R^{2}$; $O O_{1}=e=R_{2}-\left(R-\delta_{0}\right)$ и $\angle O_{1} O C=(\pi-x)$ и произведя преобразования, получим для $m$ выражение $m=\sqrt{R_{2}^{2}-\left[\left(R_{2}-R+\delta_{0}\right) \cdot \sin x\right]^{2}}-\left(R_{2}-R+\delta_{0}\right) \cdot \cos x$, которое, после подстановки в него (39), принимает вид

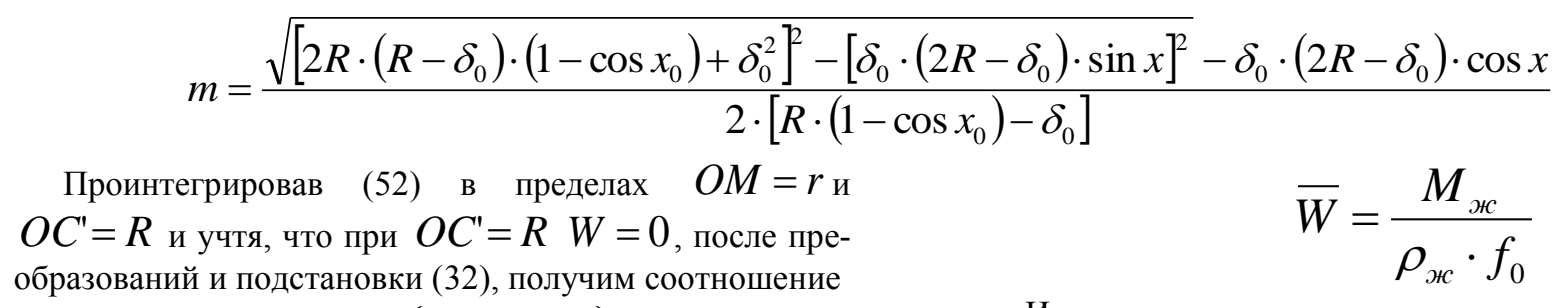
$O C^{\prime}=R$ и учтя, что при $O C^{\prime}=R W=0$, после пре-

$$
W_{(x, r)}=\frac{g \cdot \rho_{\varkappa} \cdot \sin \alpha}{\mu_{ж}} \cdot\left(\frac{R+r}{2}-m\right) \cdot(R-r),
$$

выражающее, совместно с (54), зависимость скорости жидкости в точке поперечного сечения потока от полярных координат этой точки. Поверхностное натяжение жидкости учтено здесь конфигурацией сечения, кривизна которого (рис. 4) количественно характеризуется углом раскрытия $x$ и максимальной толщиной потока $\delta_{0}$, связанные полученным ранее уравнением (51).

Выражение для максимальной скорости (в точке D) найдем при условии $x=0$, чему из (54) следует $m=R-\delta_{0}$. После преобразований получим

$$
W_{\max }=\frac{g \cdot \rho_{\varkappa} \cdot \sin \alpha}{2 \mu_{ж}} \cdot\left(\delta_{0}\right)^{2}
$$

Среднюю скорость течения определим на основе (37), выражение для которой с учетом (55), представляя двойным интегралом вида

$$
\bar{W}=\frac{2 g \cdot \rho_{\varkappa} \cdot \sin \alpha}{\mu_{\varkappa} \cdot f_{0}} \cdot \int_{0}^{x_{0} R} \int_{m}^{R} r \cdot(R-r) \cdot\left(\frac{R+r}{2}-m\right) \cdot d r \cdot d x
$$

в котором площадь живого сечения потока, являясь разностью площадей секторов $A D^{\prime} B E A$ и $A D^{\prime} B D A$, выражается как

$$
f_{0}=\frac{R^{2}}{2} \cdot\left[\left(2 x_{0}-\sin 2 x_{0}\right)-\left(\frac{\sin x_{0}}{\sin _{2}^{0}}\right)^{2} \cdot\left(2 x_{2}^{0}-\sin 2 x_{2}^{0}\right)\right]
$$

где $x_{2}^{0}$ определяется в соответствии с (50).

С другой стороны, средняя скорость выражается из уравнения сплошности, как
Из изложенного выше следует, что ручейковое течение жидкости в трубах аппаратов АХА с учетов ее поверхностного натяжения описывается замкнутой системой уравнений (51), (57), (54), (58), (50) и (59).

Эта система должна решаться с учетом ограничений, связанных с физикой процесса. Так, смачиванию жидкостью поверхности трубы, а, следовательно, вогнутости поверхности жидкости, удовлетворяет условие (рис. 4)

$$
R_{2}>R
$$

чему согласно (39) соответствует неравенство $2 R \cdot\left(R-\delta_{0}\right) \cdot\left(1-\cos x_{0}\right)+\delta_{0}^{2}>2 R \cdot\left[R \cdot\left(1-\cos x_{0}\right)-\delta_{0}\right]$ , (61)

решение, которого

$$
x_{0}<\arccos \left(\frac{\delta_{0}}{2 R}-1\right)
$$

определяет верхний предел возможных значений угла охвата полусечения $x_{0}$.

Нижний предел для значения $x_{0}$ получим из условия - значения текущего радиуса $r=m$ положительны во всем диапазоне возможных наборов пар величин $x_{0}$, $\delta_{0}$.

Этому условию согласно (54), соответствует неравенство

$$
R \cdot\left(1-\cos x_{0}\right)>\delta_{0}
$$

решая которое получим

$$
x_{0}>\arccos \left(1-\frac{\delta_{0}}{R}\right)
$$

Объединяя (62) и (64), запишем 


$$
\arccos \left(1-\frac{\delta_{0}}{R}\right)<x_{0}<\arccos \left(\frac{\delta_{0}}{2 R}-1\right)
$$

Таким образом, указанная выше система уравнений, решаемая с учетом ограничения (65), представляет собой математическую модель безнапорного ламинарного течения жидкости в трубах аппаратов AXА с учетом поверхностного натяжения. Так как интеграл в уравнении (57) в общем виде не берется, система решается численными методами.

\section{ВЫВОДЫ}

Проведенная работа позволила сформулировать следующие выводы.

1. Разработанные модели дают возможность рассчитывать основные параметры безнапорного ламинарного ручейкового течения жидкой фазы в трубах теплообменных аппаратов AXА, такие как геометрические размеры и площадь поперечного сечения потока, а также его максимальную и среднюю скорости.

2. Расчеты можно производить без учета поверхностного натяжения жидкости и, если это необходимо, с его учетом.

3. Модель с течением жидкости концентрическими слоями несколько уступает модели с неконцентрическими слоями по физическому и математическому обоснованиям, однако по точности получаемых результатом обе модели практически эквивалентны.

4. Методы, используемые в настоящем разделе, позволяют подойти к разработке модели течения жидкого аммиака в слабонаклоненных трубах испарителей AXA с внутренней насечкой, позволяя рассмотреть влияние сил поверхностного натяжения с учетом кривизны поверхности в двух взаимноперпендикулярных направлениях (формулы (41) и (42)) с радиусами $R_{2}$ и $r_{2}$, причем последней может быть связан с величинами шага и глубины насечки.

\section{ЛИТЕРАТУРА}

1. Захаров Н.Д. Новые конструкции энергосберегающих бытовых абсорбционных холодильных аппаратов / Н.Д. Захаров, А.С. Титлов, О.Б. Васылив, Д.С. Тюхай // Холодильная техника и технология. - 1998. - № 58. - С. 44-52.

2. Захаров М.Д. Аналіз ексергетичної ефективності циклів АДХМ / М.Д. Захаров, О.С. Тітлов, Д.С. Тюхай, Ю.С. Ботук, О.Б. Василів // Наукові праці Одеської державної академії харчових технологій. - 2001. - № 22. - C. 161-167.

3. Титлов А.С. Современный уровень разработок и производства бытовых абсорбционных холодильных приборов / А.С. Титлов // Холодильный бизнес. - 2007. - № 8. - C. 12-17; № 9. - С. 28-30; № 10. - С. 47-49 ; №
11. - C. 46-47.

4. Ботук Ю.С. Математическое описание процессов тепломассообмена в испарителе абсорбционнодиффузионной холодильной машины / Ю.С. Ботук, А.С. Титлов, О.Б. Васылив, Л.Р. Ленский // Тепловые режимы и охлаждение радиоэлектронной аппаратуры. Научно-технический сборник. -1998. - № 1. - С. 10-12.

5. Титлов А.С. Анализ и моделирование тепловых режимов противоточного жидкостного теплообменника абсорбционно-диффузионной холодильной машины / А.С. Титлов, Д.С. Тюхай, О.Б. Васылив // Вестник Международной академии холода. - 2002. - № 1. - С. 19-21.

6. Buz V. N. The mathematical and experimental modeling of absorption-diffusion refrigerators / V. N. Buz, M. A. Buкraba, H. F. Smirnov // Int. seminar and workshop "Heat pipes, beat pumps, refrigerators. Dual-use technologies", Sept. 15-18, 1995, Minsk, Belarus. Preprint.

7. Титлов А.С. Методика расчета трехпоточных испарителей абсорбционно-диффузионных холодильных машин / А.С. Титлов, Ю.С. Ботук, О.Б. Васылив, В.В. Завертаний // Вестник Международной Академии Холода. - 1998. - № 2. - С. 26-27.

8. Штеренлихт Д.В. Гидравлика: - В 2-х кн.: кн.2 -2-е изд., перераб. и доп.- М.: Энергоатомиздат, 1991.- 367 с. 9. Тітлов О.С. Методика розрахунку термодинамічних параметрів циклу абсорбційно-дифузійних холодильних машин (АДХМ) / О.С. Тітлов // Наукові праці Одеської державної академії харчових технологій. - 1997. № 17. - C. 272-276.

10. Титлов А.С. Экспериментальные исследования температурно-энергетических характеристик низкотемпературных камер на основе АДХМ / А.С. Титлов, В.В. Завертаний, О.Б. Васылив, Л.Р. Ленский // Тепловые режимы и охлаждение радиоэлектронной аппаратуры. Научно-технический сборник. -1998. - № 1. - С. 60-67.

11. Титлов А.С. Энергосберегающие режимы работы перекачивающих термосифонов бытовых холодильных машин абсорбционного типа / А.С. Титлов, Д.С. Тюхай // Вестник Международной академии холода. - 2000. № 4. -C. 13-15.

12. Очеретяный Ю.А. Испытания на парусной яхте абсорбционного холодильника с горелочным устройством / Ю.А. Очеретяный, С.К. Чернышов, А.С. Титлов, А.К. Ширшков // Холодильная техника и технология. 2006. - № 5. - C.49-51.

13. Кириллов В.Х. Компьютерное моделирование физических и технологических процессов. Теория, алгоритмы, программы / В.Х. Кириллов, В.В. Зуб, А.С. Титлов, А.К. Ширшков // Учебное пособие. - Одесса: Издательство ВМВ, 2016. - 565 с.

14. Богданов С.Н., Иванов О.П., Куприянова А.В. Холодильная техника. Свойства веществ -Изд. 3-е перераб. и доп. -М.: Агропромиздат, 1985. -208 с. 


\title{
Modeling of the manual non-stopped current modes of the liquid phase of the working body in the elements of absorption refrigerating devices
}

\author{
A. S. Titlov, O. B. Vasylsv, D. B. Adambaev \\ Odessa National Academy of Food Technologies, st. Kanatnaya, 112, Odessa, 65039, Ukraine
}

In the transition of household refrigeration to natural working bodies a special place occupy refrigerators with absorption refrigeration units (ARU). ARU working medium is a water ammonia solution with an inert gas (hydrogen, helium or their mixture). The operation of absorption refrigerators can be carried out from any source of thermal energy with sufficient temperature potential. ARU have no moving elements in the design, are silent and reliable in operation, their cost is minimal among analogues. The main drawback of absorption refrigerators is high energy consumption. This is due to the lack of model ideas about the processes of heat and mass transfer and flow regimes of the working fluid in the elements of the $A R U$ in the development and serial models design. It is known only one model for calculating the ARU evaporator, which includes empirical relationships borrowed from the work of the Leningrad Institute of Food and Refrigeration Industry and allows determining the average flow rate of the liquid phase and the configuration of the stream section. These relationships, however, are applicable only to pipes with a smooth inner surface, they do not take into account the influence of surface tension forces that bend the interfacial surface; besides, for these the range of parameters in which they are obtained is not given. Therefore, they should be considered as indicative and applicable only for preliminary assessments. The special literature discusses methods for calculating rippled turbulent sections characteristic of river effluents and large canals, which is not applicable for evaporator tubes and ARU absorber with diameters about 20 mm. Studies by employees of the Odessa National Academy of Food Technologies and the Vasilkovsky Refrigerator Plant showed that a laminar flow of the evaporating ammonia-coolant liquid phase takes place in the cylindrical tube of the ARU evaporator. The liquid forms a trickle moving under the action of gravity along and down the pipe located at an angle to the horizon. The forces acting on the element of a liquid coolant moving uniformly inside a smooth pipe are considered. The flow model without the surface tension of the liquid is presented. There were two ways to solve the problem. In the first method, it was assumed that the fluid flow is layered and its layers are concentric with the inner surface of the pipe. The expression obtained as a result of the simulation is the dependence of the flow rate of the fluid on the half-angle of the cross section. It is shown that constant speed lines are arcs of circles, concentric inner surface of the evaporator tube. The second way to solve the problem also involves the layering of fluid flow, however, without limitation on the location of the layers. With this method of solving the problem, the flow rate of liquid ammonia at the point of the cross section is determined by the polar coordinates of this point. Numerically, the average velocity and configuration of the cross section for both methods of determination differ slightly (within $5 \%$ ). Therefore, for practical calculations both methods can be applied. The model for calculating the flow with the surface tension forces is given. It is assumed that the curvilinear phase interface is the surface of a circular cylinder of radius. The work carried out allowed us to formulate the following conclusions. 1. The developed models make it possible to calculate the main parameters of the free-flow laminar streamlet flow of the liquid phase in ARU tubes of heat exchangers, such as the geometric dimensions and cross-sectional area of the flow, as well as its maximum and average velocities. 2. Calculations can be made without taking into account the surface tension of the fluid and, if necessary, taking it into account. 3. The model with the flow of fluid by concentric layers is somewhat inferior to the model with non-concentric layers in terms of physical and mathematical substantiation; however, in terms of the accuracy of the result, both models are almost equivalent.

Key words: absorption refrigerating units, streamed free flow, simulation, calculation, analysis

\section{REFERENCES}

1. Zakharov N.D. New designs of energy-saving household absorption refrigerators / ND Zakharov, A.S. Titlov, OB Vasilyv, D.S. Tyuhay // Refrigeration and technology. 1998. - № 58. - p. 44-52.

2. Zakharov MD Analysis of efficiency of cycles ADHM / M.D. Zakharov, O.S. Titlov, D.S. Tyuhay, Yu.S. Botuk, OB Vasiliv // Science and Science of the Odessa State
Academy of Harvest Technologies. - 2001. - № 22. - p. 161-167.

3. Titlov A.S. The modern level of development and production of household absorption refrigeration devices / A.S. Titlov // Refrigeration business. - 2007. - № 8. - p. 12-17; № 9. - p. 28-30; № 10. - p. 47-49; № 11. - p. 46-47.

4. Botuk Yu.S. Mathematical description of heat and mass transfer processes in the evaporator of an absorptiondiffusion cooling machine / Yu.S. Botuk, A.S. Titlov, OB Vasyl, L.R. Lensky // Thermal regimes and cooling of elec- 
tronic equipment. Scientific and technical collection. 1998. - № 1. - p. 10-12.

5. Titlov A.S. Analysis and modeling of thermal conditions of a countercurrent liquid heat exchanger of an absorptiondiffusion chiller / A.S. Titlov, D.S. Tyuhay, OB Vasyl // Bulletin of the International Academy of Cold. - 2002. - № 1. - p. 19-21.

6. Buz V. N. The chemical and experimental modeling of absorption-diffusion refrigerators / V. N. Buz, M. A. Bukraba, H. F. Smirnov // Int. seminar and workshop "Heat pipes, beat pumps, refrigerators. Dual-use technologies", Sept. 15-18, 1995, Minsk, Belarus. Preprint.

7. Titlov A.S. Method of calculating three-flow evaporators of absorption-diffusion cooling machines / A.S. Titlov, Yu.S. Botuk, OB Vasyliv, V.V. Wrapping // Bulletin of the International Academy of Cold. - 1998. - № 2. - p. 26-27.

8. Shterenlicht D.V. Hydraulics: - In 2 books: kn.2-2 ed., Pererab. and additional - M .: Energoatomizdat, 1991.$367 \mathrm{p}$.

9. Titov O.S. Methods of thermodynamic rosrahunkuk parametriv in the cycle of absorption-diffusion refrigerating machines (ADHM) / O.S. Titles // Naukov_ Prats_of the Odessa State Academy of Harvest Technologies. - 1997. № 17 . - p. 272-276.
10. Titlov A.S. Experimental studies of the temperature and energy characteristics of low-temperature chambers based on ADHM / A.S. Titlov, V.V. Wraps, OB Vasyl, L.R. Lensky // Thermal regimes and cooling of electronic equipment. Scientific and technical collection. -1998. - № 1. - p. 60-67.

11. Titlov A.C. Energy-saving modes of operation of pumping thermosiphons of absorption-type household refrigerating machines / A.S. Titlov, D.S. Tyuhay // Bulletin of the International Academy of Cold. - 2000. - № 4. -C. 13-15.

12. Ocheretyany Yu.A. Tests on a sailing yacht of an absorption refrigerator with a burner / Yu.A. Ocheretyany, S.K. Chernyshov, A.S. Titlov, A.K. Shirshkov // Refrigeration and technology. - 2006. - № 5. - P.49-51.

13. Kirillov V.Kh. Computer simulation of physical and technological processes. Theory, algorithms, programs / V.H. Kirillov, V.V. Tooth, A.S. Titlov, A.K. Shirshkov // Tutorial. - Odessa: Publishing of WWII, 2016. - 565 p.

14. Bogdanov S.N., Ivanov OP, Kupriyanova A.V. Refrigeration. Properties of substances - Exit. 3rd revised and add. -M .: Agropromizdat, 1985. -208 p.

Received 18 February 2018 Approved 20 March 2018 Available in Internet 30 June 2018 